\title{
Measuring the Differential Economic Impact of Education across Income Groups and Provinces in Pakistan: A Model- Consistent Approach
}

\section{Fahd Rehman* and Russel J. Cooper**}

\begin{abstract}
Engel's Law states that the share of food in household expenditure declines with households' total expenditure - a regularity that is clearly evident in Pakistani household income and expenditure data. This study uses an "Engel curve" to incorporate additional social effects-including the impact of education on welfare-to infer the differential impact of education on measures of household wellbeing across income groups and provinces. Our Engel curve specifications close the gap between economic theory and empirical applications critical to evaluating the effects of education on economic wellbeing. The results show that net primary and matriculation education enrolment ratios can bring about a significant improvement in people's welfare. Accordingly, there is a need to specifically redirect resources to Balochistan where access to educational opportunities is rather low; and to increase access to such opportunities in Sindh and Khyber Pakhtunkhwa. Data for the period 2008-11 shows that households in the two lowest income groups are worst off in terms of access to educational opportunities. Efforts should thus be stepped up to enhance their access to educational opportunities at the primary and matriculation levels. The study's predictions are intended to guide policymakers in terms of where to concentrate their efforts and reduce economic distortions, and move the economy onto a sustainable path in the long run.
\end{abstract}

Keywords: Modified Almost Ideal Demand System, Pakistan, education, hedonic prices.

JEL classification: P24, I131.

\section{Introduction}

Improvements in education indicators are central to economic development as people pay a great deal of attention to their families'

\footnotetext{
${ }^{*}$ Doctoral Economics student, School of Business, University of New South Wales, Canberra.

** Visiting Professor, School of Business, University of New South Wales, Canberra.
} 
education. Schultz (1988) shows that additional years of schooling have strong effects on an individual's enhanced earnings, and education has prime importance in sustaining income growth. Sen (1999) argues that better educated people are efficient producers and more likely to make informed choices. Bardhan and Udry (1999) hold that income inequality is the result of lack of attainment of education in poor countries. Considering the link between education and income, access to educational opportunities could prove a sound policy instrument for uplifting a beleaguered economy such as Pakistan.

Access to educational opportunities is pivotal to promoting the equitable distribution of resources among different groups in a society. One of the biggest challenges facing provincial governments in Pakistan is their inability to redirect resources to provide even coverage and access to educational opportunities to a large population (United Nations Development Programme, 2011a, 2011b). The lack of access to educational opportunities and the scarcity of existing resources in the context of a developing country such as Pakistan call for a proper diagnosis of the problem such that resources are redirected to improve people's wellbeing.

In studying these important issues, we apply econometric analysis to a model already well grounded in theory. This allows us to maximize the compatibility between our statistical estimation and the economic implications we draw. The literature that informs our research centers on the consumer demand system, especially studies that have estimated regular demand systems capable of providing the basis for robust economic analysis. Specifically, Barnett and Serlitis (2008) have conducted a survey of consumer choice in a static framework, and argue for the importance of global regularity in empirical demand systems. They also use an Engel curve in making welfare comparisons across different groups and determining the properties of demand systems. They point out that standard empirical demand systems do not provide an accurate picture of observed behavior across income groups, which can, however, be captured using an Engel curve. Additionally, Cooper and McLaren (1992) have developed a model known as the Modified Almost Ideal Demand System (MAIDS), which satisfies the global regularity condition and fits the Engel curve very well.

Much of the literature shows that there are often indirect effects as the economy benefits from growth and that these benefits pass through the circular flow of income. To capture and evaluate the full effects on household wellbeing, we use an indirect approach that measures 
consumers' wellbeing by observing their spending patterns. We then link this to various factors that contribute to access to educational opportunities by constructing a hedonic price explanator; this index is used to estimate consumption behavior.

Specifically, we base our analysis of household behavior on a decision-making model driven by households' understanding of their real expenditure position, which we think of as their effective real expenditure position or their "true" standard of living defined within a utilitymaximizing economic paradigm. The effective real expenditure position of a household is what drives its consumption behavior and, hence, underpins its Engel curve characteristics in our model. This effective real expenditure is defined as nominal expenditure divided by a householdspecific price deflator that takes into account the impact of the household's educational level on the quality of its consumption prospects. This, in turn, introduces the level of education as an explanator of the "true" (qualityadjusted) prices faced by the household.

Engel's Law, an empirical regularity observed for around 200 years, states that the share of food in household expenditure declines with households' total expenditure (income). Technically, this means that the income elasticity of demand for food is less than unity. This phenomenon is the predicted outcome of an economic model based on individual consumers' (or households') rational attempts to maximize their economic welfare (or utility) subject to a budget constraint-provided that their preferences are specified as nonhomothetic, i.e., that their preferences are different at different levels of income. Household budget data enables us to estimate an Engel curve, which is a function describing how a consumer's purchase of a particular good changes with variations in his/her income.

What is the expected effect of an improvement in living standards on consumers' food budget shares? We hypothesize that this will lead to one of two effects in terms of the Engel curve representation of consumer behavior. If the change in standard of living is reflected in the data-based measures of total real expenditure, then the consumer effectively "slides" down the food Engel curve. This would then lead to an expected, observed reduction in the food expenditure share (in favor of a greater expenditure share on "luxuries" relative to the necessity, food).

On the other hand, if the improved living standard is not immediately reflected in verifiable income changes, we will still expect the same consumer behavior even though the real income change could not be 
measured. In many cases, with data based on household expenditure surveys-particularly in developing countries such as Pakistan-reliable estimates of household income are not available, especially when economic activity takes place partly or wholly in the informal sector. We therefore need to allow for this scenario and be able to model the Engel curve for food as shifting downward with improvements in the standard of living.

To our knowledge, the development economics literature has not used the empirical regularity expressed in Engel's Law to infer measures of household wellbeing from observations on food budget shares or assessed the impact of access to educational opportunities on wellbeing in this context. The literature also fails to consider access to education as the ability to purchase things. Our study is thus a novel approach to measuring the impact of education on wellbeing by exploiting Engel's Law.

In the consumer demand literature, the application of MAIDS has been restricted mainly to individual household datasets. Here, however, we apply MAIDS to aggregate data to show that it is equally suitable for handling aggregate datasets. Another contribution of this approach is that it uses the available secondary sources with a limited amount of aggregate data to draw meaningful conclusions. Our approach makes a theoretical contribution because it combines several modern economic approaches in a manner not hitherto attempted. To our knowledge, no existing study that is fully consistent with theory combines hedonic pricing with consumer demand systems to deal with a situation where official differential prices are not available and to actually estimate and use differential effective prices.

The empirical regularity expressed in Engel's Law is clearly present in Pakistan's Household Integrated Economic Survey (HIES) datasets. However, it has not been used to infer measures of household wellbeing from observations on food budget shares; our paper addresses this gap. This is a model-consistent approach as we attempt to reduce the gap between economic theory and practice by carefully adapting the model after having studied the data and invoked the role of economic agents. In principle, our approach provides differentiated evaluations for citizens from different walks of life and who may live in different provinces. Determining the differential impact is critical to evaluating the impact of initiatives on the growth of a more cohesive and inclusive society.

Section 2 presents a theoretically consistent MAIDS model. Section 3 describes the data and statistics used. Section 4 estimates our MAIDS model and gives its results. Section 5 concludes the paper with policy recommendations and areas suggested for future research. 


\section{A Theoretically Consistent MAIDS}

of the form:

We represent household preferences by an indirect utility function

$$
\left(M / \prod_{i=1}^{N} p_{i}^{\beta_{i}}\right) \ln \left(M / \prod_{i=1}^{N} p_{i}^{\alpha_{i}}\right)
$$

where $\sum_{i=1}^{N} \alpha_{i}=1, \sum_{i=1}^{N} \beta_{i}=1$ and $M$ represent total consumer expenditure , i.e., $\left.M=\sum_{i=1}^{N} p_{i} q_{i}\right)$. Specification (1) is a special case of Cooper and McLaren's (1992) model. Applying Roy's Identity (see, for example, Barnett \& Serlitis, 2008, for details) under the neoclassical static utility-maximizing paradigm implies a consumer demand system of the form:

$$
S_{i}=\frac{\alpha_{i}+\beta_{i} \ln \left(M / \prod_{i=1}^{N} p_{i}^{\alpha_{i}}\right)}{1+\ln \left(M / \prod_{i=1}^{N} p_{i}^{\alpha_{i}}\right)}, i=1, \ldots, N
$$

where $s_{i}=p_{i} q_{i} / M$, the share of product $i$ in total expenditure $M$.

Estimating (2) completely requires nonlinear methods because of the presence of the explicit price index $P_{A} \equiv \prod_{i=1}^{N} p_{i}^{\alpha_{i}}$ in both the numerator and denominator of the fractional specification for each product share. Given, however, the limited data available-and especially the little or no direct information on price variability across our observations-we propose some carefully constructed modifications to the above scheme to aid estimation in the context of our aggregative household data set.

First, noting the unavailability of observationally differentiated data on $p_{i}$, we employ a specially constructed price index for $P_{A}$ in (2). Denoting this special index (described in Section 3) as $P_{S}$, our estimating form becomes:

$$
S_{i}=\frac{\alpha_{i}+\beta_{i} \ln \left(M / P_{S}\right)}{1+\ln \left(M / P_{S}\right)}, i=1, \ldots, N
$$

Second, provided that $P_{S}$ is constructed from exogenous data prior to estimation, we can now rearrange (3) into a convenient linear estimation form by utilizing the following nonlinear transform for real expenditure: 


$$
Z=\frac{\ln \left(M / P_{S}\right)}{1+\ln \left(M / P_{S}\right)}
$$

Note that $Z$ is bounded from above by unity. It is also possible to scale the data such that $M$ and $P_{S}$ are both normalized to unity either at or below the lowest real expenditure observation in the data. This means that $Z$ can also be bounded from below by 0 . Now, using (4), (3) can be rewritten as:

$$
s_{i}=\alpha_{i}+\delta_{i} Z \text { where } \delta_{i}=\beta_{i}-\alpha_{i}, i=1, \ldots, N
$$

This gives a very straightforward simple linear regression specification for household demands for each product share. The original parameter restrictions $\sum_{i=1}^{N} \alpha_{i}=1, \quad \sum_{i=1}^{N} \beta_{i}=1$, which imposed linear homogeneity on the price indexes in the indirect utility function, show up empirically in (5) as the cross-equation restrictions $\sum_{i=1}^{N} \alpha_{i}=1, \sum_{i=1}^{N} \delta_{i}=0$. However, as is well known for consumer demand systems, these restrictions need not be explicitly imposed but can be enforced simply by excluding one of the $N$ equations in (5) from estimation.

Third, again in response to data availability, we concentrate on household demands for two simple aggregate items-food, on the one hand, and all other products on the other. Letting the subscript $F$ denote food and the subscript $R$ denote the remaining aggregative product, we can simplify (5) further to a two-equation system:

$$
\begin{aligned}
& S_{F}=\alpha_{F}+\delta_{F} Z \quad \text { where } \delta_{F}=\beta_{F}-\alpha_{F} \\
& S_{R}=\left(1-\alpha_{F}\right)-\delta_{F} Z
\end{aligned}
$$

Note that $S_{F}$ and $s_{R}$ fully represent all budget shares at this level of aggregation, and hence $s_{F}+s_{R}=1$.

At this level of aggregation, food is a necessity. According to the well-established empirical validity of Engel's Law, we expect the share of food to fall as real income rises. Since we also expect real income to be monotonically related to our $Z$ measure, we can interpret (6) as follows. The parameter $\alpha_{F}$ represents the share of food in the budget for a very poor household - either the lowest real-income household in the sample if we normalize $M / P_{S}$ to unity for this household, or an even poorer 
household in principle if we normalize such that $M / P_{S}>1$ for the lowest income in the sample.

If one looks back to the system in (3), it is clear that $\beta_{i}$ denotes the asymptotic share-the proportion of the budget spent on product $i$ as income grows indefinitely. In terms of (6), Engel's Law suggests that $\beta_{F}<\alpha_{F}$, so that the budget share of the necessity, i.e., food, falls as $Z$ rises. Hence, $\delta_{F}<0$ for the food equation.

Figure 1 illustrates the Engel curve for food: in part (a), shown as a linear function of $Z$, and in part (b), shown as the equivalent nonlinear function of $M$.

Figure 1: Share of food as a nonlinear function of $M$

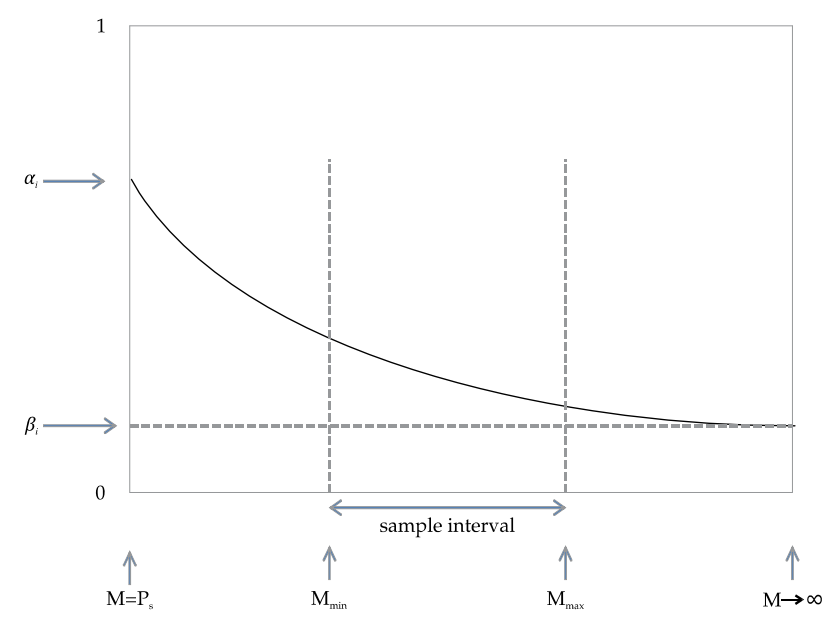

Empirically, we estimate the Engel curve for food in the form of (5), using household expenditure survey data. Since food is a necessity, we expect the general shape of the Engel curve to be as illustrated in Figure 1. However, the question that arises is whether one food Engel curve will suffice to explain the behavior of all consumers. Essentially, we want to know whether we should be estimating a different Engel curve for a subclass of members of a consumer group if those subclass members have a different degree of access to educational opportunities. We also want to know whether the results differ across provinces.

We examine these issues by constructing a different measure of $P_{S}$ depending on the degree of access that households have to educational 
opportunities. A different value of $P_{S}$ for two households with the same $M$ would mean that they face different quality-adjusted prices, due to different educational opportunities. This would be represented in Figure 1a by two different points on the curve as $Z$ differs between the households.

In terms of Figure 1b, such a difference in educational opportunities but not in nominal total expenditure would be represented by a shift in the curve. The initial point $\alpha_{F}$ and the asymptotic final point $\beta_{F}$ would not themselves change, and a "shift" in the case of Figure $1 \mathrm{~b}$ needs to be understood as a change in the degree of curvature of the nonlinear function. The essential point though is that there is a different nonlinear curve of the type given in Figure $1 \mathrm{~b}$ for households facing different educational opportunities. However, we can estimate all relevant parameters by the one linear relationship, given in Figure 1a.

\section{Data and Descriptive Statistics}

Our approach aims to provide a modeling structure that will aid informed policymaking with minimal resource requirements over the currently available data sources. For this reason, we concentrate on using household expenditure surveys, which are normally completed within a short time period to ensure that households face almost identical prices. We have, therefore, used pooled data from secondary sources such as the HIES and Pakistan Social and Living Standards Measurement (PSLM) surveys for the analysis. Both surveys are conducted under the aegis of the Pakistan Bureau of Statistics (PBS) and their questionnaire design, sampling techniques, data collection, data entry, table production, data analysis, report writing, and publication are thus consistent with each other.

We employ household expenditure data on average monthly consumption expenditure by commodity groups and quintiles for Pakistan both at an aggregate level ${ }^{1}$ and further classified by province (Punjab, Sindh, Khyber Pakhtunkhwa [KP], and Balochistan). The data contains the basic background information for the Engel curves in a simple form as the share of food items in total expenditure falls with increases in income. The sample consists of 15,512 households with an average household size of 6.58 for 2007/08 across Pakistan. The average household size shows a decreasing trend from the first to the fifth quintile, indicating that the richest households are smaller than the middle-income and poor

${ }^{1}$ Household consumption expenditure refers to all money expenditure by the household and individual members on goods intended for consumption and expenditure on services. It also includes the value of goods and services received "in kind" or "own-produced" that are consumed by the household. 
households. The average household size varies across provinces and is 6.33, 6.50, 7.63, and 7.75 for Punjab, Sindh, KP, and Balochistan, respectively (PBS, 2009a).

The average household size is 6.38 across Pakistan for 2010/11; this shows a slight reduction in average size from 2008 to 2011. The sample consists of 16,341 households and the average household size varies across provinces-6.16, 6.39, 7.17, and 7.08 for Punjab, Sindh, KP, and Balochistan, respectively (PBS, 2011a). Again, the average household size across all the provinces is slightly smaller than for 2008.

We match this data against quintiles from PSLM survey for 2007/08 and 2010/11, using provincial and income quintiles. The first quintile contains individuals with the lowest consumption level, whereas the fifth quintile contains individuals with the highest consumption level. Similarly, the net primary ${ }^{2}$ and net matriculation ${ }^{3}$ level enrolment ratios are further classified by province and quintile (PBS, 2009b, 2011b). Combining the PSLM with the HIES, which are both useful datasets, enables us to differentiate the effective prices of quality-adjusted expenditures.

\subsection{Key Educational Statistics and Pooled Data}

Table 1 presents the key educational statistics for all provinces for 2008 and 2011. On average, Punjab fares better than the other provinces in terms of net primary enrolment (NPE) and net matriculation enrolment (NME) ratios. Its NPE exceeds that of Sindh, KP, and Balochistan by 10, 12, and 20 percentage points, respectively, in 2008, and by 8, 10, and 14 percentage points in 2011. However, while Punjab's NPE indicators remain more or less constant over this period, the gap between Punjab, Sindh, KP, and Balochistan shrinks from 2008 to 2011.

All four provinces record a dismally low NME for 2008 (Table 1). Punjab leads once again, with a 13 percent enrolment rate, surpassing Sindh, KP, and Balochistan by 2, 7, and 8 percentage points. The NME gap between Punjab and Sindh remains small, but increases with reference to KP and Balochistan. Punjab's NME for 2011 increases to 14 percent, surpassing that of Sindh, KP, and Balochistan by 3, 7, and 8 percentage points. The gap between Punjab and Sindh increases slightly by 1 percentage point, but remains the same for KP and Balochistan.

2 Net primary enrolment ratio $=$ [number of children aged 5-9 years attending primary school (classes 1-5) divided by the number of children aged 5-9 years] multiplied by 100 .

${ }^{3}$ Net matriculation enrolment ratio = (number of children aged 13-14 years attending matriculation level divided by number of children aged 13-14 years) multiplied by 100 . 
Table 1: Summary of key educational statistics across provinces (percentage)

\begin{tabular}{lccrc}
\hline 2007/08 & Punjab & Sindh & KP & Balochistan \\
\hline NPE ratio & 61 & 51 & 49 & 41 \\
NME ratio & 13 & 11 & 6 & 5 \\
\hline 2010/11 & & & & \\
NPE ratio & 61 & 53 & 51 & 47 \\
NME ratio & 14 & 11 & 7 & 6 \\
\hline
\end{tabular}

Source: Pakistan Bureau of Statistics.

In order to achieve sufficient degrees of freedom and allow for some province-specific effects, we pool the data for 2008 and 2011 across all four provinces. The distributional information is available from the income quintiles and provinces. Pooling the data requires inflation-adjusted expenditure, so the expenditure data for 2008 is divided by the combined consumer price index of 100, while the data for 2011 is divided by 146.5 (constructed by the PBS). Figure 2 provides a scatter plot of food against inflation-adjusted income for 2008 and 2011.

Figure 2: Scatter plot of food against income, 2008 and 2011

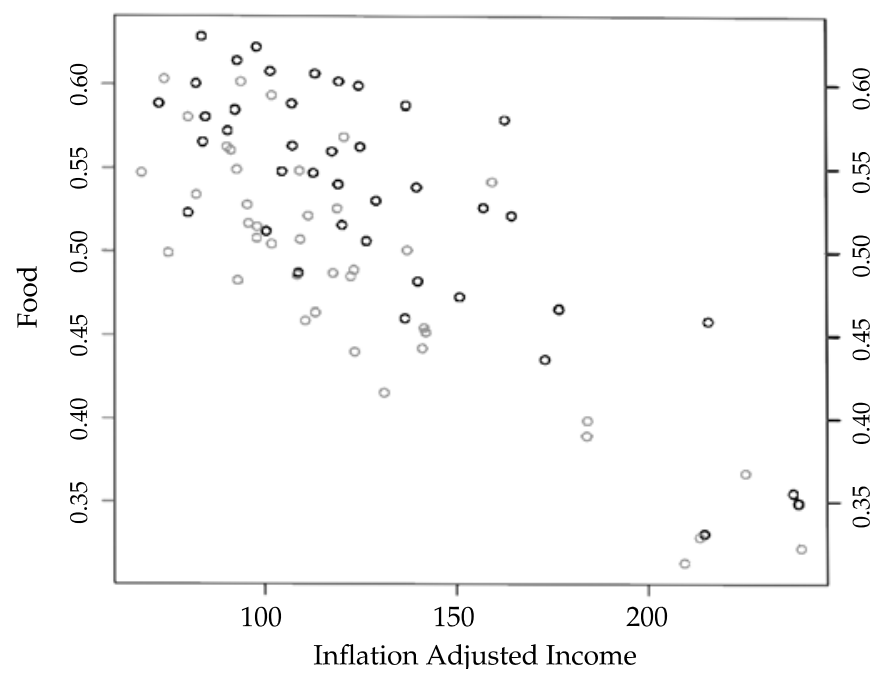

Note: Dark circles = data points for 2008, light circles = data points for 2011.

Source: Authors' calculations based on data from the Pakistan Bureau of Statistics.

This scatter plot provides very useful qualitative information. The data points for 2008 are lower than those for 2011, showing that the share of food in total expenditure increased over this period. This indicates that, 
on average, people were worse off from 2008 to 2011 as they spent more on necessities (food) and less on luxuries. We then calculate the following:

- Food ratio = food share in 2008/food share in 2011

- Income ratio = inflation-adjusted income in 2008/inflation-adjusted income in 2011

The average food ratio becomes 1.09 percent while the average income ratio is 1.05 percent. A comparison of these ratios shows that food expenditure outpaced inflation-adjusted expenditure from 2008 to 2011, indicating, on average, a decline in people's living standards in Pakistan.

The scatter plot given in Figure 2 should be compared directly with the theoretical Engel curve in Figure 1b. It is immediately apparent that, in terms of our model, the nonlinear Engel curve viewed as a function of $M$ has actually risen over the relevant years. This implied deterioration in the standard of living is something we investigate further with special interest in the differences in degree of deterioration across provinces.

The interaction plot of the mean of food and provinces against year in Figure 3 shows consumers' behavior. Generally, consumers are worse off from 2008 to 2011. It also shows the relative performance of consumers across the provinces on average: those in Balochistan are worst off, while those in Punjab remain better off than the others. One interesting feature is that consumers in Sindh appear to be even worse off than those in KP.

\section{Figure 3: Interaction plot of provinces and food against year}

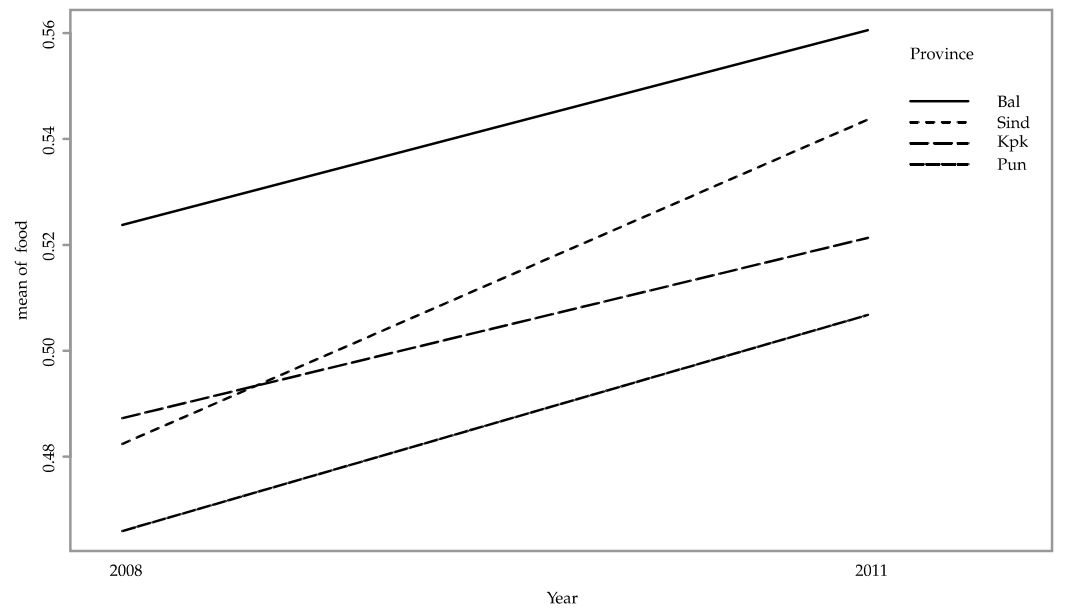

Source: Authors' calculations based on data from the Pakistan Bureau of Statistics. 
The interaction plot of the geometric means of the NPE and NME ratios and provinces against year in Figure 4 reflects the education situation. The geometric mean captures the difference in the enrolment ratios well. The interaction plot shows the relative indicators of education across the four provinces. Punjab is in a far better position than all the other provinces; Balochistan remains at the bottom. The education indicators in $\mathrm{KP}$ improve somewhat, while those in KP deteriorate slightly. These figures are a good guide to precise modeling.

\section{Figure 4: Interaction plot of provinces and geometric mean of education} against year

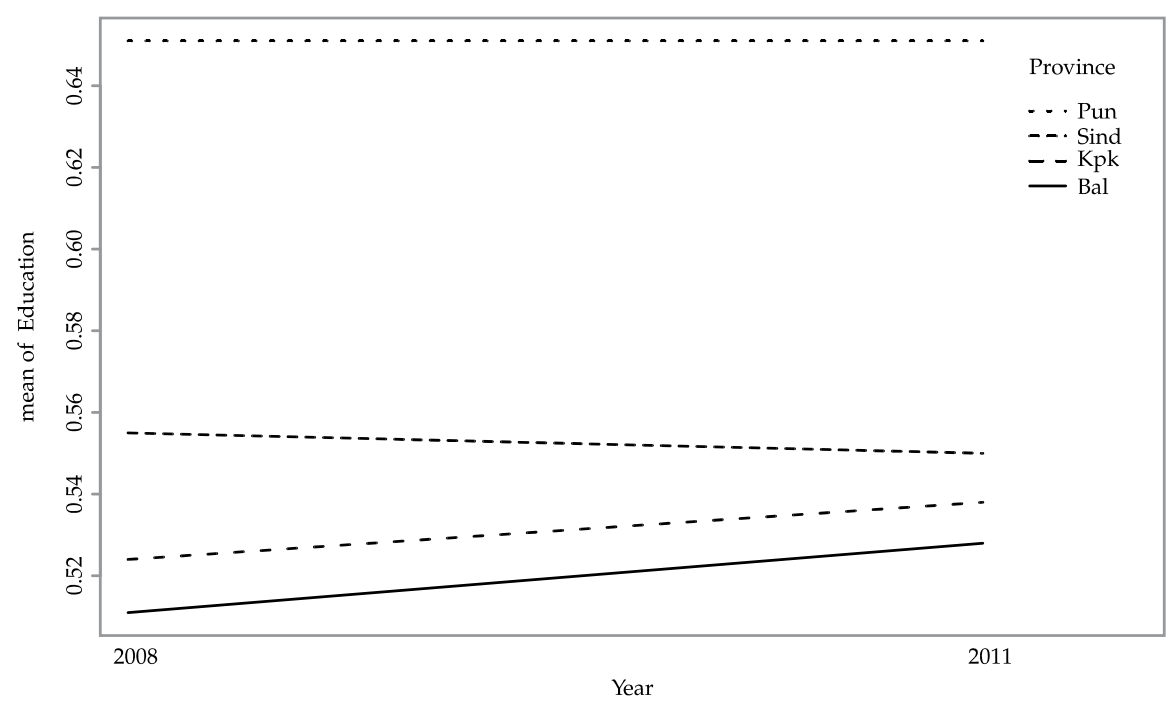

Source: Authors' calculations based on data from the Pakistan Bureau of Statistics.

\subsection{Adapting the Model to Handle Data Availability}

Our cross-sectional data does not contain information on price variability. Ideally, we want to use quality-adjusted prices, by which we mean that households with greater educational opportunities are likely to have access to higher-quality products and, thus, in quality-adjusted terms, to have the benefit of facing lower prices. We therefore use information on educational opportunities to construct a price index that is inversely related to these opportunities-the hedonic approach to pricing.

Given the Engel curve dataset with which we are working, we have assembled two possible measures of educational opportunity that match our observations: the NPE ratio and the NME ratio. NPE and NME are constructed directly from the ratios given in the PBS data and expressed as 
percentages. Normalizing the minimum levels of NPE and NME in our dataset, we construct the following simple indexes:

- $\quad$ Normalized NPE $=$ NPE - minimum $(\mathrm{NPE})$

- $\quad$ Normalized NME $=$ NME - minimum (NME)

These normalized indexes range upward from 0 and indicate increasing educational opportunity. To construct price equivalents, given that we envisage increased quality-adjusted price as being associated with less opportunity, we define two possible price indexes:

- $\quad \mathrm{PPE}=1-$ normalized NPE

- $\mathrm{PME}=1-$ normalized NME

Note that, as the raw price indexes representing the standard of living, these prices are unity for those in the provinces with the lowest level of educational opportunity. They are lower, reflecting a higher standard of living, for those households that have greater educational opportunities.

It is not clear which of these two measures would best represent the impact of educational opportunities on quality-adjusted prices. Arguably, both are somewhat relevant. Therefore, in addition to trying both alternatives, we also form a geometric mean price index for the two possible measures. This gives us our final measure of $P_{S}$.

$P_{S}=$ geometric mean price index $(P P E, P M E)=P P E^{0.5} P M E^{0.5}$

To summarize, we consider a naïve model in which we set $P_{S}=1$, denoted as Model 1. We then consider the two alternatives $P_{S}=P P E$ (Model 2) and $P_{S}=P M E$ (Model 3). Finally, $P_{S}$ constructed as in (7) is denoted as Model 4.

\section{Estimation and Results}

This section develops a series of models to consider different measures of educational opportunity. 


\subsection{The Basic Model}

Following convention, we append an additive disturbance term to the food equation given in (6). Our estimating form is:

$$
s_{F}=\alpha_{F}+\delta_{F} Z+v
$$

We make the usual assumptions that $v \sim N\left(0, \sigma^{2}\right)$. This is something of an approximation because the dependent variable is restricted to the $(0,1)$ range. However, so is the deterministic component of the model and, hence, within-sample errors can be expected to be similarly restricted. There is no a priori reason to assume the presence of large positive or negative disturbances that would violate the $(1,0)$ bound in practice, even though this is possible in principle with a normality assumption. We test for evidence on whether normality is likely to be violated. If it were, we could consider estimation under a log-ratio transformation, but this would require nonlinear estimation. We begin with a simple linear estimation of (8) by least squares and generally find that normality cannot be rejected.

As mentioned above, in order to thoroughly consider different measures of educational opportunity, we estimate several versions of the model. In the first version, which we refer to as the simple model, we ignore the effect of educational opportunities. Essentially, $P_{S}$ is set at unity for all provinces in this model. The results for 2008 are given in Table 2.

Table 2: Results of simple Engel curve for 2008

\begin{tabular}{llcc}
\hline Model 1 & $\alpha$ & $\delta$ & $\beta$ \\
\hline & $0.63^{* * *}$ & $-0.44^{* * *}$ & $0.19^{* * *}$ \\
R-squared & 0.59 & & \\
Breusch-Pagan p-value & 0.84 & & \\
Shapiro-Wilk normality p-value & 0.54 & & \\
\hline
\end{tabular}

Note: ${ }^{* * *}=$ significant at 1 percent.

The estimate of the $\alpha$ intercept is 0.63 , while the slope, $\delta$, is -0.44 . We calculate the $\beta$ coefficient to be 0.19 as $\beta_{F}=\delta_{F}-\alpha_{F}(\beta=\delta-\alpha)$. Since food is a necessity, $\alpha$ is greater than $\beta$, which is consistent with our earlier proposition. Moreover, the $\alpha$ coefficient measures the intercept of the Engel curve, while the $\beta$ coefficient measures the asymptotic value of the food share as expenditure increases along the expenditure axis. The Breusch- 
Pagan and Shapiro-Wilk tests are used to check the robustness of the results, and give no evidence of heteroskedasticity or nonnormality.

\subsection{Developing the Model}

The simple Model 1 is then adapted to handle the availability of data as explained in Section 3. This yields Model 2, which takes into account the effect of NPE on food share. The NPE ratio enters the equation as the hedonic price index and converts nominal expenditure into real expenditure. The real expenditure variable describes the movement along the Engel curve. The estimates of $\alpha$ and $\beta$ are 0.67 and 0.24 , respectively, and the model satisfies the diagnostic criteria. Model 3 caters for the effect of NME on food share. $\alpha$ and $\beta$ are 0.65 and 0.22 , respectively, and the model satisfies the robustness criteria stated above.

The coefficients $\alpha$ and $\beta$ in Models 2 and 3 have useful implications. By introducing the NPE ratio in Model 2 as the hedonic price index for education, the movement along the Engel curve is captured by the $\beta$ coefficient. The curve also shifts upward in economic terms as in Figure 1, as measured simultaneously by $\alpha$ and $\beta$. This hedonic price index also shows that education improves real expenditure, which can be called quality-adjusted expenditure.

Similarly, the NME ratio is introduced in Model 3 as the hedonic price index, and the Engel curve moves slightly upward as depicted by $\alpha$ and $\beta$. The values of the coefficients imply that the NPE ratio has a smaller effect on welfare than the NME ratio in 2008. This welfare improvement is depicted by an improvement in households' real expenditure through education. These results are intuitive: additional years of schooling enhance welfare and those households with greater access to educational opportunities are better off.

In Model 4, the education variable enters the equation via the price index $P_{S}$ in the form of the hedonic geometric mean price index (7). Again, this turns nominal into real expenditure. Through this model, we attempt to capture the joint effect of the NPE and NME ratios, but with one advantage: it captures both effects with a single geometric mean price index. The values of $\alpha$ and $\beta$ are 0.66 and 0.23 , respectively, which fall in between those of Model 2 and Model 3 (see Table 3). 
Table 3: Results with hedonic price index for 2008

\begin{tabular}{llcc}
\hline Model 2 & \multicolumn{1}{c}{$\alpha$} & $\delta$ & $\beta$ \\
\hline NPE & $0.67^{* * *}$ & $-0.43^{* * * *}$ & $0.24^{* * *}$ \\
R-squared & 0.66 & & \\
Breusch-Pagan p-value & 0.95 & & \\
Shapiro-Wilk normality p-value & 0.28 & & \\
\hline Model 3 & & & \\
NME & $0.65^{* * *}$ & $-0.43^{* * *}$ & $0.22^{* * *}$ \\
R-squared & 0.66 & & \\
Breusch-Pagan p-value & 0.76 & & \\
Shapiro-Wilk normality p-value & 0.31 & & \\
\hline Model 4 & & & \\
Geometric mean price index & $0.66^{* * *}$ & $-0.43^{* * *}$ & \\
R-squared & 0.67 & & \\
Breusch-Pagan p-value & 0.78 & & \\
Shapiro-Wilk normality p-value & 0.18 & & \\
\hline
\end{tabular}

Note: ${ }^{* * *}=$ significant at 1 percent.

The basic results of the simple Engle curve for 2011 are shown in Table 4 . The estimate of the $\alpha$ intercept is 0.65 , while the slope $\delta$ is -0.38 . Again, the relationship between $\alpha$ and $\delta$ becomes hyperbolic as already shown in Figure 1, so the slope is negative. We calculate the $\beta$ coefficient to be 0.27 as $\beta_{F}=\delta_{F}-\alpha_{F}(\beta=\delta-\alpha)$. Food is a necessity so $\alpha$ is greater than $\beta$, which is consistent with our earlier assumption. The Breusch-Pagan and Shapiro-Wilk tests are used to check the robustness of the model at a conventional significance level, and do not indicate any heteroskedasticity or nonnormality.

Table 4: Results of simple Engel curve for 2011

\begin{tabular}{llcc}
\hline Model 1 & \multicolumn{1}{c}{$\boldsymbol{\alpha}$} & \multicolumn{1}{c}{$\boldsymbol{\delta}$} & $\boldsymbol{\beta}$ \\
\hline & $0.65^{* * *}$ & $-0.38^{* * *}$ & $0.27^{* * *}$ \\
R-squared & 0.48 & & \\
Breusch-Pagan p-value & 0.092 & & \\
Shapiro-Wilk normality p-value & 0.58 & & \\
\hline
\end{tabular}

Note: ${ }^{* * *}=$ significant at 1 percent.

Similarly, Model 2 shows the effect of the NPE ratio on food share in 2011 (Table 5). The NPE ratio enters the equation as the price index and converts nominal into real expenditure. The estimates of $\alpha$ and $\beta$ 
coefficients are 0.69 and 0.32 , respectively, and the model satisfies the diagnostic criteria. Model 3 caters for the effect of NME on food share. The $\alpha$ and $\beta$ coefficients are 0.67 and 0.28 , respectively, and the model satisfies the diagnostic testing procedure at a conventional level of significance.

In Model 4, the education variable enters the equation via the price index $P_{S}$ in the form of the hedonic geometric mean price index (7), again converting nominal into real expenditure. This model helps us estimate the joint effect of the NPE and NME ratios. The values of $\alpha$ and $\beta$ are 0.68 and 0.30, respectively, which fall in between those of Model 2 and Model 3. The results are given in Table 5 .

Comparing the values of $\alpha$ and $\beta$ for 2008 and 2011 across Model 4 indicates the clear upward movement of the Engel curve after incorporating the effect of access to educational opportunities. $\alpha$ moves upward from 0.66 to 0.68 and $\beta$ moves even further, from 0.23 to 0.30 . This is a substantial shift upward and slightly flattens the Engel curve. However, this model needs to be refined to address two underlying issues. First, inflation needs to be adjusted from 2008 to 2011. Second, we are interested in the differential impact across income groups and provinces, and this leads to the models shown in Tables 6 and 7.

Table 5: Results with hedonic price index for 2011

\begin{tabular}{llcc}
\hline Model 2 & \multicolumn{1}{c}{$\alpha$} & $\delta$ & $\boldsymbol{\beta}$ \\
\hline NPE & $0.69^{* * *}$ & $-0.37^{* * *}$ & $0.32^{* * *}$ \\
R-squared & 0.53 & & \\
Breusch-Pagan p-value & 0.12 & & \\
Shapiro-Wilk normality p-value & 0.19 & & \\
\hline Model 3 & & & \\
NME & $0.67^{* * *}$ & $-0.39^{* * *}$ & $0.28^{* * *}$ \\
R-squared & 0.55 & & \\
Breusch-Pagan p-value & 0.09 & & \\
Shapiro-Wilk normality p-value & 0.61 & & \\
\hline Model 4 & & & \\
Geometric mean price index & $0.68^{* * *}$ & $-0.38^{* * * *}$ & \\
R-squared & 0.54 & & \\
Breusch-Pagan p-value & 0.11 & & \\
Shapiro-Wilk normality p-value & 0.28 & & \\
\hline
\end{tabular}

Note: ${ }^{* *}=$ significant at 1 percent. 
Model 5 adapts Model 4 by applying it to the pooled dataset. Table 6 gives the results of inflation-adjusted pooled data further deflated by the quality-adjusted geometric mean price index for education. The model shows quality-adjusted expenditure to measure the true cost of living. We constrain the values of the $\beta$ coefficient for 2008 and 2011 on the assumption that wealthy households will have similar access to educational opportunities across different provinces and times; the estimate of $\beta$ is 0.26 . We allow the value of the $\alpha$ coefficient to change from 2008 to 2009 through the introduction of a time dummy. The result shows that the time dummy is statistically significant and positive. Model 5 satisfies the diagnostic criteria at a conventional level of significance of 5 percent.

Table 6: Results for pooled data with time dummy variable

\begin{tabular}{lcccc}
\hline Model 5 & $\alpha$ & $\delta$ & $\beta$ & Time dummy \\
\hline & $0.65^{* * *}$ & $-0.39^{* * *}$ & $0.26^{* * *}$ & $0.08^{* * *}$ \\
R-squared & & 0.63 & & \\
Breusch-Pagan p-value & & 0.36 & & \\
Shapiro-Wilk normality p-value & & 0.08 & & \\
\hline
\end{tabular}

Note: ${ }^{* * *}=$ significant at 1 percent.

The time dummy variable takes a value of 0 for 2008 and 1 for 2011. The purpose of adding a time dummy variable is to determine the differential economic impact of education across different income groups. The time dummy variable adds to the $\alpha$ coefficient, while the $\beta$ coefficient remains unchanged as it is deliberately constrained. The positive magnitude of the time dummy variable reflects the upward movement of the Engel curve, suggesting that households in the two lowest quintiles were worse off in terms of access to educational opportunities over this period. The quantitative results corroborate the earlier qualitative evidence in Figure 2.

\subsection{Differential Economic Impact of Education across Provinces}

Model 6 in Table 7 is an adaptation of Model 5 in Table 6 . We construct provincial dummy variables for the pooled data, which take the value of 0 for 2008 and 1 for 2011. The year 2008 is considered the base period and the relative performance of the geometric mean educational indicators is measured across the provinces for 2011. Again, the value of the $\beta$ coefficients is constrained on the assumption that wealthy households have similar access to educational opportunities across different provinces. 
The estimates of $\alpha$ and $\delta$ are 0.65 and -0.39 , respectively, and the model satisfies the diagnostic criteria as shown in Table 7.

Table 7: Results of differential economic impact of education on provinces through hedonic prices

\begin{tabular}{lccccccc}
\hline Model 6 & $\boldsymbol{\alpha}$ & $\boldsymbol{\delta}$ & $\boldsymbol{\beta}$ & $\begin{array}{c}\text { Punjab } \\
\text { dummy }\end{array}$ & $\begin{array}{c}\text { Sindh } \\
\text { dummy }\end{array}$ & $\begin{array}{c}\text { KP } \\
\text { dummy }\end{array}$ & $\begin{array}{c}\text { Balochistan } \\
\text { dummy }\end{array}$ \\
\hline Education & $0.65^{* * *}$ & $-0.39^{* * *}$ & $0.26^{* * *}$ & 0.03 & $0.10^{* * *}$ & $0.08^{* * *}$ & $0.11^{* * *}$ \\
$\begin{array}{l}\text { R-squared } \\
\text { Breusch- }\end{array}$ & 0.66 & & & & & \\
$\begin{array}{l}\text { Pagan p-value } \\
\begin{array}{l}\text { Shapiro-Wilk } \\
\text { normality p- }\end{array}\end{array}$ & 0.59 & & & & & \\
value & 0.15 & & & & & \\
\hline
\end{tabular}

Note: ${ }^{* * *}=$ significant at 1 percent.

The results obtained through this model capture quality-adjusted expenditure against food for 2011. Since the price index for access to educational opportunities deflates inflation-adjusted expenditure, this again becomes a hedonic approach to prices. We observed that the food share increased from 2008 to 2011 in Figure 2, and Model 6 highlights the economic impact of education across the provinces through the provincial dummies. Apart from Punjab, all the provincial dummies turn out to be positive and highly significant. Their positive magnitudes suggest the upward movement of the $\alpha$ coefficient.

Access to educational opportunities decreases from 2008 to 2011 in the case of Balochistan; the coefficient of the provincial dummy is larger and positive. Households in Sindh are worse off, as shown by its coefficient, followed by those in KP. This implies that households in Sindh and Balochistan are worse off since 2008 in terms of access to educational opportunities. Although the coefficient of the Punjab dummy remains positive, it is statistically insignificant, suggesting that access to educational opportunities remains almost the same from 2008 to 2011.

\section{Conclusion, Policy Recommendations, and Future Areas of Research}

Our approach contributes to the theoretical literature because it combines several modern economic approaches in a manner not hitherto attempted. Specifically, the existing literature does not combine hedonic pricing with consumer demand systems to deal with the situation where official differential prices are not available, and to actually estimate the 
differential economic impact across income groups and provinces in a theoretically consistent manner by invoking the role of economic agents.

These results are consistent with the finding that additional years of schooling enhance individuals' income earnings. We contribute to this by according greater significance to education, considering it the ability to purchase things, i.e., households with greater access to educational opportunities are better off. The behavior of economic agents further implies increasing uncertainty in the economy over the period of study.

The upward movement of the Engel curve from 2008 to 2011 shows that, on average, households were worse off. The NME ratio has a significantly greater impact than the NPE ratio. The results also show that households across the two lowest income quintiles were worse off from 2008 to 2011 in terms of access to educational opportunities. Similarly, the upward movement of the Engel curve across different provinces shows that households in Balochistan, Sindh, and KP were worse off, while Punjab remained unaffected.

\subsection{Policy Recommendations}

Differential access to educational opportunities across income groups and provinces calls for astute policymaking. Based on our findings, we recommend the following measures:

1. Households that fall in the two lowest income groups were worse off in terms of access to educational opportunities from 2008 to 2011. Efforts should be stepped up to enhance access to educational opportunities at the primary and matriculation levels across these lowest-income groups. This could play a significant role in reducing the rising educational inequality, which, in turn, could lead to income inequality over a period of time.

2. There is a need to specifically redirect resources to Balochistan where access to educational opportunities at the primary and, specifically, the matriculation level is rather low. Access to educational opportunities should also be increased in Sindh and KP.

3. The simple Engel curve moved upward from 2008 to 2011, which is a reliable indicator of the increase in poverty because the consumption of food in total expenditure increased. Efforts should thus be made to reduce the level of poverty. 
4. As the impact of the NME ratio is significantly greater than that of the NPE ratio, efforts should be made to increase enrolment rates at the matriculation level.

5. The sampling frames for urban areas were updated in 2003 while those for rural areas were based on the population census of 1998 for HIES data. These need to be upgraded such that the sampling frames for urban and rural areas are consistent enough to obtain wellinformed predictions.

\subsection{Future Areas of Research}

We have estimated the food Engel curve for 2008 and 2011 across income groups, but we have not provided money-metric measures of welfare differentials due to the uneven socioeconomic distribution of educational opportunities across income groups. The provision of rigorously derived measures of the compensating and equivalent variations (as defined in Hicksian welfare analysis) from 2008 to 2011 for various income groups and in different provinces could be a future area of research.

Although we have estimated the differential economic impact of education across income groups and provinces, there is a need to extend this modeling structure across districts and rural and urban regions, which would require data at the quintile and district level. This could prove a possible area of research by employing individual household datasets. Moreover, the average household size varies from 2008 to 2011, implying that further demographic extensions are possible for estimation and inference by looking closely at the individual household data. 


\section{References}

Bardhan, P., \& Udry, C. (1999). Development microeconomics. New York, NY: Oxford University Press.

Barnett, W. A., \& Serlitis, A. (2008). Measuring consumer preferences and estimating demand systems. In D. Slottje (Ed.), Quantifying consumer preferences (Contributions to economic analysis, vol. 288, pp. 1-31). Bingley, UK: Emerald Press.

Cooper, R. J., \& McLaren, K. R. (1992). An empirically oriented demand system with improved regularity properties. Canadian Journal of Economics, 25(3), 652-668.

Pakistan Bureau of Statistics. (2009a). Household integrated economic survey 2007-08. Islamabad, Pakistan: Author.

Pakistan Bureau of Statistics. (2009b). Pakistan social and living standards measurement survey 2007-08. Islamabad, Pakistan: Author.

Pakistan Bureau of Statistics. (2011a). Household integrated economic survey 2010-11. Islamabad, Pakistan: Author.

Pakistan Bureau of Statistics. (2011b). Pakistan social and living standards measurement survey 2010-11. Islamabad, Pakistan: Author.

Schultz, T. P. (1988). Education investments and returns. In H. Chenery \& T. N. Srinivasan (Eds.), Handbook of development economics (vol. 1). Amsterdam: Elsevier Science Publisher.

Sen, A. (1999). Development as freedom. New York, NY: Alfred A. Knopf.

United Nations Development Programme. (2011a). Balochistan millennium development goals report 2011. Islamabad, Pakistan: Author.

United Nations Development Programme. (2011b). Punjab millennium development goals report 2011. Islamabad, Pakistan: Author. 